\title{
20. Langage II
}

\author{
(c) Springer-Verlag France 2010
}

\section{2}

\section{La démence sémantique}

S. Belliard ${ }^{1,2}$, C. Merck ${ }^{1}$, P.Y. Jonin ${ }^{1}$

${ }^{1}$ CMRR, Rennes

${ }^{2}$ Inserm U923, Caen

La démence sémantique (DS) est un syndrome d'atrophie focale décrit en 1989 et lié à une atrophie temporale antérieure asymétrique. Elle apparaît généralement entre 50 et 70 ans mais des formes plus tardives sont possibles.

Le tableau se caractérise par des troubles sémantiques au premier plan, c'est à dire des troubles multimodaux d'identification des objets et des personnes. Le patient ou son entourage se plaignent de ne pas comprendre certains mots, de ne pas reconnaître certaines personnes. Cette perte sémantique peut, au départ, épargner certains items, en particulier ceux que le patient rencontre souvent ou qui ont un lien direct avec sa biographie (notion d'égocentrisme cognitif). Des troubles du caractère et du comportement apparaissent secondairement. Le patient est décrit comme psychorigide, égocentrique. Les autres capacités cognitives sont préservées pendant plusieurs années.

La DS est souvent considérée comme une forme d'aphasie progressive (la forme fluente) même si le trouble cognitif dépasse largement le cadre du langage et s'il existe d'autres formes d'aphasie progressive fluente. Elle est souvent classée au sein des dégénérescences lobaires frontotemporales avec l'aphasie progressive non fluente et la démence frontotemporale.

Initialement, la DS était considérée comme une entité non Alzheimer, c'est-à-dire dont les lésions histologiques sous jacentes étaient toujours différentes de celles que l'on rencontre dans la maladie d'Alzheimer. Il est vrai que le tableau est liée dans la grande majorité des cas à l'accumulation d'une protéine anormale, la protéine la TDP43, mais certains travaux récents montrent que dans une minorité de cas (moins de $20 \%$ ), la DS peut être liée à une authentique maladie d'Alzheimer histologique.
20.3

\section{Principes de la prise en charge orthophonique des troubles du langage d'origine dégénérative D. Perrier-Palisson \\ Orthophoniste, CMRR centre, CHRU de Tours}

Les troubles du langage sont fréquents chez le sujet âgé et l'aphasie, secondaire à des lésions vasculaires, est le trouble le plus classiquement rééduqué depuis de nombreuses années. La prise en charge des pathologies du langage d'origine neurodégénérative s'est développée plus récemment, en s'intéressant d'abord aux patients atteints de la maladie d'Alzheimer (MA) ayant un trouble prédominant dans la sphère langagière puis aux patients atteints d'une aphasie primaire progressive (APP) ou d'une démence sémantique (DS).

De nombreux travaux concernant la rééducation de l'aphasie vasculaire ont montré son caractère bénéfique et l'amélioration des capacités de communication des sujets rééduqués. À l'inverse, il existe encore actuellement peu de travaux publiés dans la littérature, confortant l'intérêt d'une telle démarche dans les pathologies neurodégénératives.

La prise en charge est souvent limitée par la coexistence de troubles cognitifs associés aux troubles du langage et par la présence de troubles sensoriels concernant la vue ou l'audition, la fatigabilité, sans oublier la motivation du patient.

Toutefois, le principe de rééducation ne doit pas être remis en cause chez le sujet âgé, à condition de respecter quelques principes de base et ne pas envisager son déroulement en plaquant les techniques utilisées dans les aphasies vasculaires. En effet, quelle que soit la pathologie, il faut garder à l'esprit que la rééducation concerne un trouble linguistique involutif, nécessitant une réévaluation régulière, afin d'adapter au mieux les aides proposées. Dans la MA, le rôle de l'orthophoniste est souvent centré sur les stratégies permettant d'améliorer la communication entre le patient et son entourage. Dans l'APP (fluente ou non fluente), l'installation lente et progressive de troubles isolés du langage, dans le contexte d'une bonne préservation de l'autonomie, rend la rééducation incontournable du fait de la conscience aiguë des troubles du langage et de la grande motivation de ces patients envers la rééducation. Enfin, la thérapie des troubles sémantiques, dans la DS, peut aussi être envisagée afin de faciliter l'accessibilité et la production d'un stock restreint de mots, propres à chaque patient, en fonction de ses centres d'intérêt et de ses difficultés au quotidien.

Quelle que soit la pathologie rééduquée, la durée de la prise en charge sera soumise au contrat thérapeutique initial que l'orthophoniste devra établir avec le patient et l'aidant principal, afin de pouvoir interrompre les séances lorsque celles-ci ne seront plus bénéfiques. 